AperTO - Archivio Istituzionale Open Access dell'Università di Torino

\title{
MORPHEUS: AN AUTOMATED TOOL FOR UNBIASED AND REPRODUCIBLE CELL MORPHOMETRY
}

\section{This is a pre print version of the following article:}

Original Citation:

\section{Availability:}

This version is available http://hdl.handle.net/2318/1738050

since 2022-12-01T08:49:49Z

Published version:

DOI:10.1002/jcp.29768

Terms of use:

Open Access

Anyone can freely access the full text of works made available as "Open Access". Works made available under a Creative Commons license can be used according to the terms and conditions of said license. Use of all other works requires consent of the right holder (author or publisher) if not exempted from copyright protection by the applicable law. 
4 Federico Alessandro Ruffinatti ${ }^{1 *}$, Tullio Genova ${ }^{2,3 *}$, Federico Mussano ${ }^{2}$ \& Luca Munaron ${ }^{3 \S}$

$5 \quad{ }^{1}$ Department of Pharmaceutical Sciences, University of Eastern Piedmont, Novara, Italy

$6 \quad{ }^{2}$ CIR Dental School, Department of Surgical Sciences, University of Turin, Turin, Italy

$7 \quad{ }^{3}$ Department of Life Sciences and Systems Biology, University of Turin, Turin, Italy

$9 \quad$ *These authors equally contributed; ${ }^{5}$ Corresponding author

11 Keywords: cell morphometry; reproducibility; orientation analysis; Fiji; ImageJ.

$13 \S$ Correspondence to:

14 Luca Munaron, Ph.D.

15 Dept. Life Sciences \& Systems Biology

16 University of Torino

17 Via Accademia Albertina 13

1810123 Torino

19 ITALY

20 luca.munaron@unito.it 


\section{ABSTRACT}

Here we present MORPHEUS, a new Fiji/ImageJ2 plugin for the automated evaluation of cell morphometry from images acquired by fluorescence microscopy. MORPHEUS works with sampling distributions to learn-in an unsupervised manner and by a nonparametric approach-how to recognize the cells suitable for subsequent analysis. Afterwards, the algorithm performs the evaluation of the most relevant cell-shape descriptors over the full set of detected cells. Optionally, also the extraction of nucleus features and a double-scale analysis of orientation can be performed. The whole algorithm is implemented as a one-click procedure, thus minimizing the user's intervention. By reducing biases and errors of human origin, MORPHEUS is intended to be a useful tool to enhance reproducibility in bioimage analysis.

\section{INTRODUCTION}

Cell morphometry can be defined as the quantitative description of cells' shape, which may not be separated from the inference of important information about their functional state. This shape-function paradigm aims to correlate cell morphology to biological processes such as proliferation, differentiation, migration, growth dynamics, adhesion to particular substrates or transition from physiology to pathology (Lepekhin et al., 2001; Ruffinatti et al., 2013; Yang et al., 2015; Lyons et al., 2016). Although many shape descriptors have been proposed over the years, no general rules about their usage currently exist since their suitability depends on the particular experimental aim (Chen et al., 2012; Lobo et al., 2015). More generally, cell morphometry lacks standardized protocols for the comparison of studies from independent laboratories. Specifically, when cells are manually selected, a heavy operator-dependent bias is introduced, impairing procedure reproducibility (Chen et al., 2016; Van Valen et al., 2016). In addition, since many samples are needed for reaching a good statistical power, human errors unavoidably arise because of the repetitiveness of such a time-consuming task. 
(ImageJ2) (https://imagej.net/ImageJ2/; https://fiji.sc/) called MORPHEUS (Multipametric Morphometric Analysis of EUcaryotic cellS). It combines within a single structured pipeline the evaluation of the most relevant cell-shape descriptors, the extraction of nucleus features and an advanced analysis of orientation. The procedure we propose is highly automated and adaptive since the full algorithm is indeed implemented in a one-click and user-friendly fashion. MORPHEUS is freely available for download at https://sourceforge.net/projects/morpheus-for-fiji/, together with the same sets of test images used in Supplementary Materials for experimental validation (see below).

\section{METHODS}

\section{Sample Preparation}

The following recommendations are made in order to generate MORPHEUS-compliant images.

1. Cells stained for some cytoplasmic element (e.g. phalloidin for cytoskeleton) need to be acquired by fluorescence microscopy. Cells stained for nuclei (e.g. through 4',6diamidino-2-phenylindole aka DAPI) are optional but not sufficient alone.

2. Images should be of good quality. If the Signal-to-Noise Ratio (SNR) is too low (i.e. less than 10), the segmentation process will possibly fail (see Section 1.2 in Supplementary Materials for an operational definition of SNR).

3. Both 8 and 16-bit gray scale images can be used, but MORPHEUS segmentation procedure always assumes light objects (fluorescence signal) on dark background. Different color lookup tables (LUTs) are not suitable for MORPHEUS.

4. Moderate and homogeneous plating densities are required. In particular, they ought to be low enough to provide a majority of isolated cells compared to cell clusters. It 
is warmly suggested to ensure even distribution of adherent cells by repetitive pipetting.

5. Since all cell clusters are automatically excluded by the pipeline, images are not to contain many pronounced cell elongations leading to cell-cell contacts (i.e. neuron networks analysis or quantitative assays of dendritic branching and arborization). Other platforms are specifically tailored for this aim (Ventimiglia et al., 1995; Wu et al., 2004).

6. The number of cells per field should be large enough to produce low-skewness sampling distributions for the statistics of interest (see below), otherwise MORPHEUS will undergo an inaccurate learning step. Hence, the use of high magnification objectives, leading to less than $10-15$ cells per field, is discouraged.

The above limits are not expected to prevent the use of MORPHEUS in a great variety of morphometric analysis contexts.

\section{Algorithm Flow}

Preliminary Steps

MORPHEUS can be easily integrated into Fiji Plugins menu by copying Morpheus_.ijm file into the appropriate directory. When launched, a dialog window allows the user to set few basic parameters (Figure 1a), among which an anti-spot noise threshold $\varepsilon$ and a tolerance value $T$ (the larger $T$, the more inclusive the cell selection will be). After pressing OK button, no further intervention is required by the user. As a preliminary task, MORPHEUS scans the input folder searching for the images to be analyzed and then it performs a segmentation in five steps: contrast enhancement, background subtraction, smoothing, thresholding and binarization (Figure 1b). Please refer to Supplementary Materials for more details about the segmentation algorithm and the other options of the starting dialog window. 


\section{Unsupervised Learning Step}

As Fiji Analyze Particles function selects the objects to be measured primarily on the basis of their area $(A)$ and their circularity $(C)$, a suitable range for these two parameters can be defined to discriminate the objects of interest (i.e. the isolated cells) from the other entities detected by the segmentation (i.e. small debris and big cell-clusters). MORPHEUS fulfils this task by analyzing the distributions of the two random variables $A$ and $C$ over the entire population and learning from the dataset the interval within which most of the isolated cells are likely to fall.

Even under controlled plating conditions, the overall distribution of $A$ is not easily predictable from a theoretical point of view. This is due both to the intrinsic biological variability among cells and, as already mentioned, to the presence of different subpopulations of objects within the same cell culture: spot noise, cellular and extracellular-matrix debris (corresponding to the high frequencies near the origin of the histogram of areas), isolated cells (the central body of the distribution) and cell clusters of two or more cells (the heavy right tail of the distribution; Figure 1d).

First, spot noise is removed by imposing a threshold $\varepsilon$ at the beginning of the algorithm (a justification for the default value $\varepsilon=200$ pixel $^{2}$ is provided in Supplementary Materials), then Analyze Particles function evaluates $A$ and $C$ for all the segmented objects that are larger than $\varepsilon$ (Figure 1c). To cope with presence of sparse cell clusters, central tendency for $A$ should be measured by an estimator robust to outliers - the median being the simplest one-that can be thought to be more representative of the single cell then the cell clusters (Figure 1d). Clearly, this is true only under the initial hypothesis of low-density plating (point 4 of Sample Preparation section), meaning that the majority of cells are indeed isolated cells. After the computation of the object median area $M_{A, i}$ for each sample image $(i=1,2, \ldots, m)$, the sampling distribution of the sample medians can be drawn (Figure 1e). This distribution, albeit not normal, is generally far less skewed than that of the starting 
population, provided the sample size is large enough (i.e. at least $10-25$ objects per image, depending on the dispersion of the overall population, as detailed in Statistical Validation section of the Supplementary Materials). By explicitly drawing such a sampling distribution from the experimental data, both the mean and the dispersion of the random variable $M_{A}$ can be evaluated in a direct way, thus providing a reliable estimate of the population median area together with its accuracy or standard error (SE). This procedure leads to the definition of a 'characteristic' or 'typical' single cell area $\left(\bar{M}_{A}\right)$ and its uncertainty $\left(\mathrm{SE}_{\bar{M}}\right)$. Starting from these assumptions,

$$
A_{\text {high }}=2 \cdot\left(\bar{M}_{A}+T \cdot \mathrm{SE}_{\bar{M}}\right)
$$

is a conservative estimate for the predicted typical size of a two-cell cluster. In other words, if an object has $A>A_{\text {high }}$, it can be legitimately suspected to be a complex of two (or more) cells. According to a log-symmetric criterion, the lower bound can be defined as

$$
A_{\text {low }}=\frac{1}{2} \cdot\left(\bar{M}_{A}-T \cdot \mathrm{SE}_{\bar{M}}\right)
$$

and all the objects below this limit will be discarded as cell debris (or residual spot noise). Depending on the tolerance value $T$ selected from the main window of MORPHEUS, the width of $\left[A_{\text {low }}, A_{\text {high }}\right]$ range can be changed, allowing for a more permissive $(T \rightarrow 6)$ or more strict $(T \rightarrow 1)$ approach to cell selection.

As for the area, also the circularity generally exhibits a complex distribution, possibly made of several subpopulations, since cell clusters can originate very low $C$ values, while debris and spot noise typically have $C$ values very close to 1 . Even in this case, MORPHEUS' approach is based on sampling distributions, albeit with some important difference compared to area determination. The multiplicative factor 2 , used to predict the expected size of a two-cell cluster, is clearly meaningless when dealing with circularity and there are no other obvious multiplicative factors able to predict the value of $C$ for those objects differing from an isolated cell. For this reason, a more conservative approach is adopted, the only aim being that of trimming the outliers. By evaluating circularity 
extreme values for each of the $m$ sample images, MORPHEUS can draw the two sampling distributions of sample minimum $\left(\min _{C}\right)$ and sample maximum $\left(\max _{C}\right)$. Again, these two distributions can be conveniently described by their mean $\left(\overline{\min }_{C}, \overline{\max }_{C}\right)$ and related SEs. Finally, by tuning the $T$ parameter, it can be set the range of circularity values to which single cells are actually supposed to belong, namely:

$$
\begin{aligned}
C_{\text {low }} & =\overline{\min }_{C}+(\tau-T) \cdot \mathrm{SE}_{\overline{\min }}, \\
C_{\text {high }} & =\overline{\max }_{C}-(\tau-T) \cdot \mathrm{SE}_{\overline{\max }},
\end{aligned}
$$

Where the constant $\tau=6$ corresponds to the highest confidence level the user can choose for parameter estimation in the current implementation of MORPHEUS (see details in Section 1.1 of the Supplementary Materials).

Once esteemed, $\left[A_{\text {low }}, A_{\text {high }}\right]$ and $\left[C_{\text {low }}, C_{\text {high }}\right]$ intervals are fed as constraints to Analyze Particles for a second run of the function over the whole dataset (Figure 1f). In other words, they are used to define a sort of bivariate confidence interval for the area and the circularity of the true isolated cell, but with no reference to any underlying distribution parameter. MORPHEUS represents indeed a completely nonparametric approach to cell morphometry, since no normality assumption is made for any of the distributions considered. Importantly, the anti-spot threshold value $\varepsilon$ and the tolerance discrete level $T$ are the only two parameters the user has to choose, thus maximally reducing the arbitrariness of the procedure and all the ensuing possible biases. In particular, the effects produced by different choices of the tolerance level have been examined and reported in Table SM3 of Supplementary Materials (Experimental Validation - Tolerance Effect section).

\section{Morphometry and Orientation Analysis}

Using the $(A, C)$-bivariate confidence interval as a filter, MORPHEUS can recognize isolated cells in each sample image (Figure 1g). Here the algorithm forks (Figure 1h): the first branch-always 
performed-is dedicated to cell morphometry, while the second one-optional-is devoted to orientation analysis. For the morphometric task, the idea is to use a wide descriptor spectrum to capture as many features as possible, preferring to reduce the dimensionality of the problem during the follow-up analysis rather than to choose a particular descriptor 'a priori' (see Figure $1 \mathrm{i}$-j and Experimental Validation - Morphometry section in Supplementary Materials). In particular, 12 different shape descriptors are evaluated for each detected cell, encompassing all those indexes natively provided by Fiji/ImageJ through the Set Measurements function (i.e. area, perimeter, best fitting ellipse (BFE) major axis, BFE minor axis, BFE aspect ratio, BFE angle, circularity, roundness, solidity, Feret's diameter, Feret's angle, and minimum caliper diameter, as detailed in Section 1.3 of the Supplementary Materials).

For the second task, MORPHEUS leans on OrientationJ, a well-established Fiji/ImageJ plugin for orientation and isotropy characterization (Rezakhaniha et al., 2012; Püspöki et al., 2016). MORPHEUS checks for OrientationJ (version $\geq 2.0 .2$ ) and, if present, OrientationJ Distribution function is called for an automated directional analysis of the whole dataset over two distinct scales: cytoskeleton and whole-cell (Figure 1k). The analysis is performed locally by means of a sliding Gaussian window with an arbitrary radius $\sigma$ that is meant to be as close as possible to the structure of interest, namely $\sigma=1$ pixel for fine structure (cytoskeleton) analysis and

$$
\sigma=\sqrt{\frac{\bar{M}_{A}}{\pi}}
$$

for the whole-cell level, taking advantage of the 'typical cell area' as estimated in the initial learning step. For both the levels of analysis, MORPHEUS returns different outputs of both qualitative and quantitative nature. In particular, two colormaps are saved carrying the directional information according to a Hue-Saturation-Brightness (HSB) color model, where hue encodes for the local dominant orientation, saturation encodes for the coherency and brightness is based on the gray 
190

191

192

193

194 195

levels of the input image (Figure 1l). In addition, a set of coherency-weighted orientation histograms are collected and then assembled into a single heatmap (Figure $1 \mathrm{~m}$ ).

\section{MORPHEUS LOg}

With the aim of being as compliant as possible with the reproducibility standards in bioinformatics (see e.g. Sandve et al., 2013), MORPHEUS prints an onscreen log at runtime and saves a copy of it in the output directory. Both the onscreen log and log file contain all the information needed to reproduce that particular analysis at a later time (see Supplementary Materials for details).

\section{RESULTS}

To have a thorough experimental validation of MORPHEUS, the interested reader can refer to the Experimental Validation section of the Supplementary Materials, in which a detailed analysis of a prototypical MORPHEUS output is presented applying the algorithm to four different original datasets of fluorescent cell samples.

\section{DISCUSSION}

Replication of experimental results at all scales of biological sciences is a pillar of scientific method. However, the crisis of reproducibility or replication crisis has been largely debated on both generalist and more specialized journals in the last years (Aleksandra et al., 2018; An, 2018; Baker, 2016; Begley \& Ellis, 2012; Coiera et al., 2018; Fanelli, 2018; França \& Monserrat, 2018; Samsa \& Samsa, 2019; Sandve et al., 2013). Different and variable factors contribute for this deep limitation in modern wet biology as well as bioinformatics fields: among them, low statistical power, poor analytic approaches and a lack of standardization in experimental protocols are unfortunately quite usual in preclinical and clinical research papers. 
According to such an increasing requirement for quality assessment and robustness,

MORPHEUS is a promising proposal for the standardization of cell morphometric analysis. Importantly, the automation descending from the adaptive nature of the algorithm eliminates the user-bias factor and translates into a reproducibility-oriented approach to cell morphometry. This is intended to avoid all those human errors typically arising from a repetitive and time-consuming task, as well as the systematic bias and arbitrariness introduced by the experimenter whenever a threshold needs to be chosen or several objects are to be recognized by sight. A general outcome of the algorithm presented in this paper lies in the intriguing inversion of the usual time spent by the operator to obtain the final results. Indeed, in the canonical 'manual' approach most of the time is dedicated to the morphometric analysis of any single frame, leading to a significant limitation in the number of processed images. Otherwise, since MORPHEUS dramatically speeds up the analysis (virtually instant output), the operator will hopefully devote most of the experimental effort in image acquisition, thus increasing the quality and the statistical power of the work.

\section{REFERENCES}

Aleksandra, T., George, L., Velocity, H. H., \& Jonas, R. (2018). Statistical mistakes and how to avoid them lessons learned from the reproducibility crisis. Osteoarthritis and Cartilage, 1-3.

An, G. (2018). The Crisis of Reproducibility, the Denominator Problem and the Scientific Role of Multi-scale Modeling. Bulletin of Mathematical Biology, 80(12), 3071-3080.

Baker, M. (2016). Is there a reproducibility crisis? Nature, 533(7604), 452-454.

Begley, C. G., \& Ellis, L. (2012). preclinical cancer research. Nature, 483, 531-533.

Chen,S. et al. (2012) Recent advances in morphological cell image analysis. Comput. Math. Methods. Med., 2012, 101536.

Chen, C. L., Mahjoubfar, A., Tai, L. C., Blaby, I. K., Huang, A., Niazi, K. R., \& Jalali, B. (2016). Deep Learning in Label-free Cell Classification. Scientific Reports, 6(March), 1-16.

Coiera, E., Ammenwerth, E., Georgiou, A., \& Magrabi, F. (2018). Does health informatics have a replication crisis? Journal of the American Medical Informatics Association, 25(April), 963-968.

Fanelli, D. (2018). Opinion: Is science really facing a reproducibility crisis, and do we need it to? Proceedings of the National Academy of Sciences, 115(11), 2628-2631.

França, T. F., \& Monserrat, J. M. (2018). Reproducibility crisis in science or unrealistic expectations? EMBO 
Reports, 19(6), e46008.

Lepekhin, E. A., Walmod, P. S., Berezin, A., Berezin, V., \& Bock, E. (2001). Evaluation of Cell Morphology. In R. H. Gavin (Ed.), Cytoskeleton Methods and Protocols (pp. 85-100). Totowa, NJ: Humana Press.

Lobo, J., See, E. Y.-S., Biggs, M., \& Pandit, A. (2016). An insight into morphometric descriptors of cell shape that pertain to regenerative medicine. Journal of Tissue Engineering and Regenerative Medicine, 10(7), 539-553.

Lyons, S. M., Alizadeh, E., Mannheimer, J., Schuamberg, K., Castle, J., Schroder, B., ... Prasad, A. (2016). Changes in cell shape are correlated with metastatic potential in murine and human osteosarcomas. Biology Open, 5(3), 289-299.

Püspöki, Z., Storath, M., Sage, D., \& Unser, M. (2016). Transforms and Operators for Directional Bioimage Analysis: A Survey. In Springer (Ed.), Focus on Bio-image informatics (pp. 69-93).

Rezakhaniha, R., Agianniotis, A., Schrauwen, J. T. C., Griffa, A., Sage, D., Bouten, C. V. C., ... Stergiopulos, N. (2012). Experimental investigation of collagen waviness and orientation in the arterial adventitia using confocal laser scanning microscopy. Biomechanics and Modeling in Mechanobiology, 11(3-4), 461-473.

Ruffinatti FA, Gilardino A, Lovisolo D, Ferraro M. Spatial wavelet analysis of calcium oscillations in developing neurons. PLoS One. 2013;8(10):e75986.

Samsa, G., \& Samsa, L. (2019). A Guide to Reproducibility in Preclinical Research. Academic Medicine, 94(1), 47-52.

Sandve, G. K., Nekrutenko, A., Taylor, J., \& Hovig, E. (2013). Ten Simple Rules for Reproducible Computational, 9(10), 1-4.

Van Valen, D. A., Kudo, T., Lane, K. M., Macklin, D. N., Quach, N. T., DeFelice, M. M., ... Covert, M. W. (2016). Deep Learning Automates the Quantitative Analysis of Individual Cells in Live-Cell Imaging Experiments. PLoS Computational Biology, 12(11), 1-24.

Ventimiglia, R., Jones, B. E., \& Møller, A. (1995). A quantitative method for morphometric analysis in neuronal cell culture: unbiased estimation of neuron area and number of branch points. Journal of Neuroscience Methods, 57(1), 63-66.

Wu, C.-C., Reilly, J. F., Young, W. G., Morrison, J. H., \& Bloom, F. E. (2004). High-throughput Morphometric Analysis of Individual Neurons. Cerebral Cortex, 14(5), 543-554.

Yang W, Tian R, Xue T. Nuclear shape descriptors by automated morphometry may distinguish aggressive variants of squamous cell carcinoma from relatively benign skin proliferative lesions: a pilot study. Tumor Biol. 2015;36(8):6125-31.

Funding information: The work was supported by a grant from the University of Turin

Conflict of interest: the authors declare no conflicts of interest

Author contribution: FAR designed Morpheus protocol and wrote the manuscript. TG performed the experiments. FM wrote the manuscript. LM designed the protocol and wrote the manuscript.

Data Availability Statement: MORPHEUS is freely available for download at https://sourceforge.net/projects/morpheus-for-fiji/, together with the same sets of test images used in Supplementary Materials for validation. 